\title{
Aprovechando el Poder de las Feromonas para Mejorar la Eficiencia Energética en Redes Definidas por Software
}

\author{
Raúl Sánchez Romero, Jaime Galán-Jiménez \\ Departamento de Ingeniería de Sistemas Informáticos y Telemáticos \\ Universidad de Extremadura \\ Cáceres, España \\ Email: rsanchezzq@alumnos.unex.es, jaime@unex.es
}

\begin{abstract}
Resumen-En los últimos años, la conciencia por la eficiencia energética se ha instalado de lleno en el seno de la sociedad. Aunque la comunidad investigadora ha realizado grandes esfuerzos en proponer soluciones para reducir el consumo de energía de las redes de comunicaciones, la mayoría de estas propuestas están pensadas para redes IP, para las cuales es necesaria la coordinación entre los distintos elementos que las componen. La aparición de las redes SDN (Software-Defined Networking) y el desacople del plano de datos del plano de control abre nuevos caminos para proponer algoritmos energéticamente eficientes a ejecutar por parte de un elemento lógicamente centralizado, el controlador SDN. En este artículo se propone un algoritmo basado en el comportamiento de las colonias de hormigas que permite la elección óptima, en términos de eficiencia energética, de los modos de operación de los enlaces de la red. Los resultados obtenidos tras su ejecución sobre topologías de red reales indican que es posible conseguir un ahorro de energía significativo, en torno al $30 \%$ para el caso en que la función de energía de los enlaces de la red sea de tipo lineal.

Palabras Clave-Eficiencia energética, SDN, ACO, nivel de energía, función de energía.
\end{abstract}

\section{INTRODUCCIÓN}

La conciencia por la mejora de la eficiencia energética ha supuesto un cambio genérico en la mentalidad de la sociedad a lo largo de los últimos años. Varios estudios indican que el consumo de energía de las TIC (Tecnologías de la Información y las Comunicaciones) supone de entre el $2 \%$ al $10 \%$ del consumo de energía a nivel mundial [1], [2]. Este consumo energético está directamente relacionado con la generación en torno al $2 \%$ del total de emisiones de $\mathrm{CO}_{2}$ a la atmósfera [1]. Además, se prevé que la demanda de energía del sector TIC crezca de forma más rápida que la demanda de energía global [3].

Por su parte, las redes de comunicaciones suponen un $37 \%$ del total de la energía requerida por la industria TIC a nivel mundial [4], [5], siendo el equipamiento de red el principal responsable del consumo de Internet [6].
Para solventar el problema del consumo de energía en las redes de comunicaciones, la comunidad investigadora ha realizado grandes esfuerzos en proponer soluciones que permitan conseguir un ahorro de energía significativo, especialmente en periodos de tiempo en los que la actividad disminuye [7]. Partiendo de la idea básica de utilizar el mínimo conjunto de elementos de red necesarios para satisfacer la demanda de tráfico dada, se han propuesto esquemas que utilizan las técnicas Sleeping y Rate Adaptation para este propósito [6]. El tráfico de la red se redigirá para cada par origen-destino por un determinado conjunto de caminos determinados de tal forma que el consumo de la red sea mínimo y no influya negativamente sobre el rendimiento de la misma.

Sin embargo, la aplicación de estas soluciones energéticamente eficientes no es sencilla a priori debido a la naturaleza distribuida de las redes IP. La filosofía centralizada del nuevo paradigma de interconexión de redes denominado redes definidas por software (SDN, Software-Defined Networking) abre nuevas oportunidades para proponer algoritmos que permitan minimizar el consumo energético global de la red. La separación del plano de datos del plano de control y la visión global del estado de la red por parte del controlador, facilita la interacción entre éste y los nodos de la red (switches SDN) ya sea para realizar consultas específicas (ej. carga de los enlaces) o actuar sobre los elementos de la red (poner a dormir un enlace o activarlo).

En este trabajo se propone un algoritmo que aprovecha la naturaleza del comportamiento animal para seleccionar la configuración óptima de una red SDN en términos de eficiencia energética. En lugar de modificar dinámicamente los caminos por los que debe viajar el tráfico desde un origen hacia un destino determinados para conseguir una reducción en el consumo de energía de la red, la solución propuesta aprovecha los fundamentos 
del algoritmo de optimización por colonia de hormigas (ACO, Ant Colony Optimization), donde lo que se persigue en cada momento es seleccionar el nivel de energía más apropiado para cada uno de los enlaces, de modo que se satisfaga la matriz de tráfico dada.

Se consideran diferentes modelos de consumo energético para los enlaces de la red (lineal, logarítmica) con el objetivo de extraer bajo qué condiciones es más apropiado utilizar uno u otro tipo. Se han realizado un conjunto de pruebas sobre topologías de red reales de distinto tamaño, cuyos resultados indican que es posible conseguir un significativo ahorro de energía en un tiempo razonable. De este modo, se demuestra que es factible instalar el algoritmo propuesto en un controlador SDN, ya que el tiempo requerido para realizar los cálculos e instalar las reglas correspondientes al encaminamiento resultante de la optimización no es considerablemente elevado.

El resto del artículo se describe como sigue. La Sección II describe los trabajos relacionados con el presentado en este artículo. El problema que se pretende resolver se describe formalmente en la Sección III, mientras que algoritmo basado en ACO para la minimización del consumo de energía en redes SDN se explica en la Sección IV. La Sección V analiza los resultados obtenidos tras las pruebas realizadas para, finalmente, indicar un conjunto de conclusiones en la Sección VI.

\section{TRABAJOS RELACIONADOS}

Durante la última década, tanto la industria como la comunidad académica han realizado grandes esfuerzos para enfrentarse al problema del consumo de energía en redes de comunicaciones. Comenzando con la idea básica de minimizar el número de recursos de red activos, se puede conseguir una reducción significativa del consumo energético en periodos de baja actividad o al redireccionar el tráfico hacia caminos específicos de modo que el resto de nodos y enlaces de la red puedan pasar a un estado de baja energía (dormido) [4], [8]. Cuando haya variaciones significativas, se deberá tomar la decisión de activar parte de estos elementos con el objetivo de satisfacer la demanda de tráfico y no afectar el rendimiento de la red.

En un paso más, las nuevas características de eficiencia energética proporcionadas por los dispositivos de red actuales abren la oportunidad de adaptar su consumo de energía a la carga de tráfico. La intención detrás de la propuesta del estándar EEE (Energy-Efficient Ethernet) fue la de reducir el consumo de energía, como mínimo, al 50\% y mantener la compatibilidad requerida con el equipamiento de red existente [9]. EEE se basa en la técnica Rate Adaptation [6], que considera distintos perfiles de consumo para el equipamiento de red con extensiones de eficiencia energética. Con esta aproximación, es posible regular el tráfico en función del perfil energético específico para cada dispositivo de la red con el objetivo final de minimizar el consumo de energía global de la red. En [10], los autores de este artículo realizan una comparación entre los dos esquemas mencionados anteriormente (Sleeping y Rate Adaptation) para proporcionar un valor concreto al número de niveles de energía que es necesario implementar en las tarjetas de línea para conseguir un ahorro de energía significativo en la red. En concreto, se establece un valor de 4 niveles de energía como máximo para conseguir un ahorro significativo ya que, un valor mayor en el número de niveles de energía apenas se traduce en incrementos significativos.

Las redes SDN definen un nuevo paradigma donde se cambia por completo la filosofía tradicionalmente implementada por las redes IP [11]. Existen dos planos claramente diferenciados: el plano de datos y el plano de control. El primero está constituido por los switches SDN, que se limitan a redirigir los paquetes entrantes en base a la información contenida en sus tablas de flujos. Estas tablas de flujos están constituidas por un conjunto de entradas del tipo $\{$ match, action $\}$ que indican la acción a realizar definida en action (ej. reenviar el paquete por un determinado puerto de salida) si se cumple el predicado definido en match (ej. paquete que ha sido enviado por un determinado nodo origen en dirección hacia otro nodo destino). Sin embargo, la lógica de control está implementada en el controlador, elemento clave del plano de control. Se trata de un dispositivo centralizado que dispone en todo momento del conocimiento global de la red y puede actuar según una lógica implementada en un algoritmo instalado en su interior. Por tanto, la aparición de las redes SDN abre nuevos caminos para proponer soluciones centralizadas energéticamente eficientes que se basen en el conocimiento adquirido por el controlador [12], [13].

Este trabajo propone una solución energéticamente eficiente para redes SDN que se basa en el comportamiento colaborativo de las hormigas (ACO). Mediante la definición de conceptos como nivel de energía y función de energía, las hormigas de la colonia representarán configuraciones de red que vendrán identificadas por el conjunto de modos de operación de los enlaces de la red. Estas soluciones potenciales deberán ser evaluadas para comprobar si finalmente son válidas en términos de un correcto encaminamiento al satisfacer la demanda de tráfico de la red. En la siguiente sección se define formalmente el problema de optimización al que se da solución en este trabajo.

\section{Definición DEL Problema}

Esta sección define y formaliza el problema de optimización que se pretende resolver con este trabajo. En concreto, el objetivo principal es minimizar el consumo de energía de una infraestructura de red SDN. Para ello, se deben definir previamente un conjunto de conceptos que serán utilizados cuando se realice el proceso de optimización.

\section{A. Formulación del Problema}

Consideremos una infraestructura de red SDN $G=$ $(V, E)$, compuesta por un conjunto de nodos $v \in V$ conectados entre sí por un conjunto de enlaces unidireccionales $e_{(i, j)} \in E$. Cada enlace unidireccional $e_{(i, j)} \in E$ que conecta el nodo $i \in V$ con el nodo $j \in V$ tiene un 


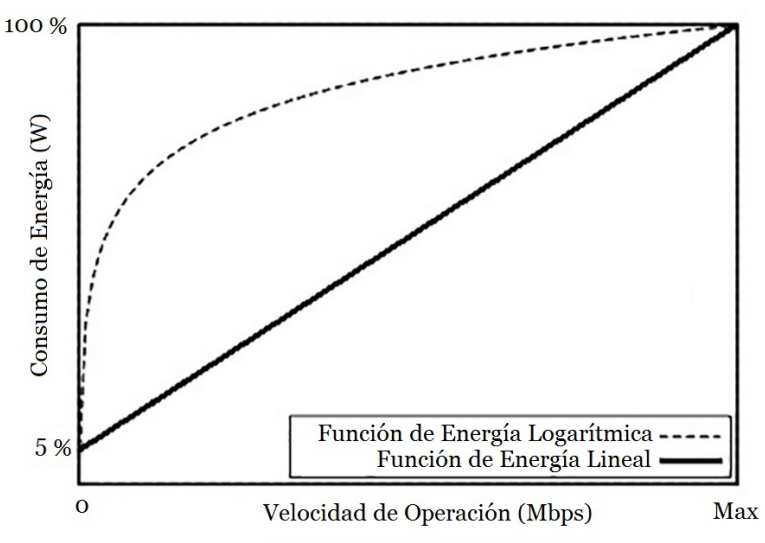

Fig. 1: Caracterización de las funciones de energía.

perfil de consumo de energía $p_{e_{(i, j)}} \in P$ y es capaz de operar utilizando $n e_{(i, j)}$ niveles de energía distintos. Se considera, además, una matriz de demanda de tráfico $M_{(i, j)}, \forall i, j \in V$ con una demanda de tráfico de $d_{(s, d)} \in$ $M_{(i, j)}$ unidades desde el nodo origen $s \in V$ hasta el nodo destino $d \in V$.

Un enlace unidireccional se denota como $e_{(i, j)} \in E$ va dirigido desde el nodo $i \in V$ hacia el nodo $j \in V$ y se encuentra operando según un nivel de energía $n e_{e_{(i, j)}} \in$ $N$. Por tanto, el objetivo es reducir el consumo de energía de la red, Eq. (1), en base a la minimización del consumo de energía de cada uno de los enlaces que la componen:

$$
\begin{aligned}
& \operatorname{minIPC}(C)=\sum_{e_{(i, j)} \in E} f_{e_{(i, j)}}\left(e_{(i, j)}, n e_{e(i, j)}\right), \\
& \forall i, j \in V, \forall e_{(i, j)} \in E, \forall n e_{e_{(i, j)}} \in N
\end{aligned}
$$

donde $f_{e_{(i, j)}}\left(e_{(i, j)}, n e_{e_{(i, j)}}\right)$ calcula el consumo de energía en Vatios del enlace unidireccional $e_{(i, j)}$, que se encuentra configurado con un nivel de energía $n e_{e_{(i, j)}}$. El consumo de energía instantáneo $I P C$ de una configuración de red $C \in G$ viene determinado por el sumatorio del consumo de energía de cada enlace, el cual depende del nivel de energía en el que se encuentre configurado.

\section{B. Definición de Nivel de Energía}

Dada una función de energía $f_{e_{(i, j)}}$, se considera nivel de energía al par $(x, y)$, donde $x$ corresponde a la velocidad de operación del enlace e $y$ es el resultado de aplicar la función de energía $f_{e_{(i, j)}}$, a $x$, con $f_{e_{(i, j)}}(x)=y$. De este modo, un nivel de energía relaciona la velocidad de operación del enlace con su consumo energético asociado. El número mínimo de niveles de energía que puede soportar un enlace $e_{(i, j)}$ es 1 o siempre activo $\left(n e_{e_{(i, j)}}=1\right)$, $\mathrm{y}$ hace referencia a la capacidad nominal del enlace. Se trata por tanto de la situación en la que no existen características de eficiencia energética. El método basado en hardware Sleeping añade otro posible nivel de energía al que poder configurar un enlace $\left(n e_{e_{(i, j)}}=2\right)$. En este caso, los enlaces pueden encontrarse o bien dormidos o bien operando a su máxima velocidad. Los enlaces que se encuentran dormidos contribuyen al ahorro de energía al no transportar paquetes de datos, aunque permanecen operativos para ser despertados si es necesario. En este nivel de baja energía, los enlaces consumen un pequeño porcentaje, que suele ser entre el 5-10 \% del consumo asociado a su nivel de energía máximo (capacidad nominal del enlace). Si por el contrario los enlaces pudiesen adaptar su velocidad de operación a la variación de la carga de tráfico a lo largo del tiempo (estándar EEE), estaríamos ante el caso de la aplicación de la técnica Rate Adaptation $\left(n e_{e_{(i, j)}}>2\right)$. En concreto, en este trabajo suponemos que los enlaces de la red pueden soportar un número determinado de niveles de energía diferentes.

\section{Caracterización de las Funciones de Energía}

Si denotamos $C_{e_{(i, j)}}$ a la capacidad nominal del enlace $e_{(i, j)} \in E$, la función de energía $f_{e_{(i, j)}}$ se define Eq. (2)

$$
\begin{aligned}
& f_{e_{(i, j)}}: C_{e_{(i, j)}} \rightarrow w_{e_{(i, j)}} \\
& \forall x \in N \exists y \in w_{e_{(i, j)}} \mid(x, y) \in N f_{e_{(i, j)}}
\end{aligned}
$$

donde $w_{e_{(i, j)}}$ es la imagen de $f_{e_{(i, j)}}$, es decir, el valor del consumo instantáneo en función de la capacidad en la que opera el enlace $e_{(i, j)}$. En la Fig. 1 se muestran las 2 funciones de energía consideradas en este trabajo: i) Lineal o proporcional, $f_{e_{(i, j)}(x)}=x$, representa una función de energía tomada como función base en la que a mayor velocidad de operación, mayor consumo de energía experimentado y ii) Logarítmica, $f_{e_{(i, j)}(x)}=\log (x)$, similar a la implementada por el estándar EEE.

\section{OPTIMIZACIÓN POR COLONIA DE HORMIGAS PARA EL AHORRo DE ENERGía}

La metaheurística basada en Colonias de Hormigas ACO (Ant Colony Optimization) abarca un conjunto de técnicas de optimización inspiradas en el comportamiento colectivo, donde el comportamiento de las hormigas está dirigido hacia la supervivencia de la colonia como un todo que al de un simple componente individual de la colonia [14]. El algoritmo ACO es una técnica probabilística utilizada comúnmente para resolver problemas computacionales que pueden ser reducidos a la búsqueda de caminos óptimos en grafos. Es en esencia un algoritmo constructivo donde en cada iteración, cada hormiga construye una solución al problema recorriendo el propio grafo. En concreto, nuestra propuesta se basa en las variantes ACS (Ant Colony System) o Sistema de Hormigas [15], [16] y en la variante Sistemas de Hormigas Elitistas, EAS (Elitist Ant System) [17].

\section{A. Codificación de la Hormiga}

La hormiga artificial (individuo de la población) es un componente computacional simple que intenta construir soluciones válidas (una posible configuración de red) al problema de coste mínimo explorando los rastros de feromona disponibles (indica la afluencia que han tenido los movimientos por anteriores hormigas) y la información heurística (como el atractivo o la mejor conveniencia de los movimientos). Una determinada solución podría no ser válida, con lo que sufrirían cierta penalización. Una solución, $s$ Eq. (3), es representada por una sucesión de niveles de energía, $n e_{e_{(i, j)}}$, donde cada uno de ellos 


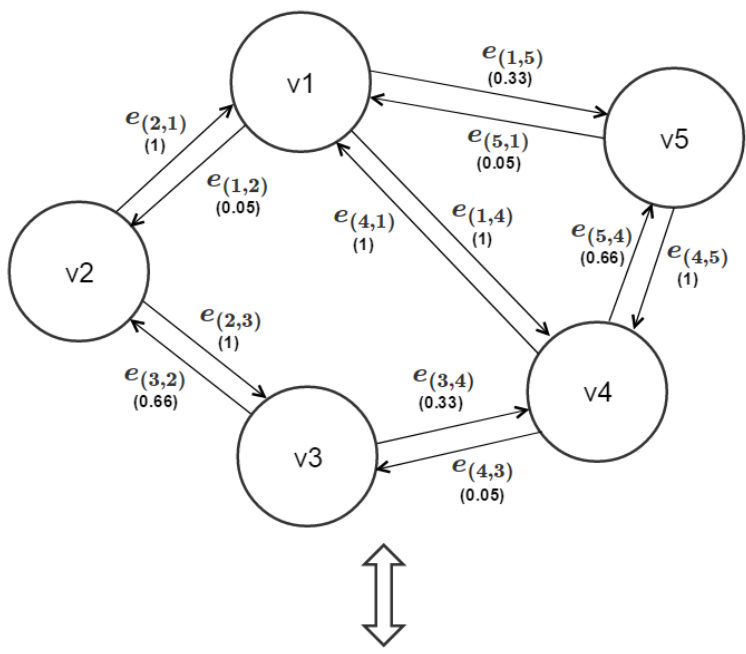
$e_{(1,2)} e_{(1,4)} e_{(1,5)} e_{(2,1)} e_{(2,3)} e_{(3,2)} e_{(3,4)} e_{(4,1)} e_{(4,3)} e_{(4,5)} e_{(5,1)} e_{(5,4)}$
\begin{tabular}{|c|c|c|c|c|c|c|c|c|c|c|c|}
\hline 0.05 & 1 & 0.33 & 1 & 1 & 0.66 & 0.33 & 1 & 0.05 & 0.66 & 0.05 & 1 \\
\hline
\end{tabular}

Fig. 2: Ejemplo solución de una hormiga con función de energía lineal y cuatro niveles de energía.

representa el nivel de energía en el que operará cada enlace de la red, $e_{(i, j)}$, identificado en $s$ por $i$.

$$
s=\left\{n e_{0}, n e_{1}, n e_{2}, \ldots, n e_{|E|}\right\}, \forall n e_{i} \in N, \forall i \in E
$$

Por tanto, cada hormiga dentro del algoritmo tendrá por objetivo obtener una configuración de red ponderando para cada enlace de la misma el nivel de energía con el que va a operar. Para ello, la hormiga va construyendo la solución iterativa e incrementalmente recorriendo todos los enlaces de la red (de menor a mayor identificador de nodo origen).

En la Fig. 2 se muestra el ejemplo de una configuración de red como solución de una hormiga considerando una topología de red compuesta por 5 nodos y 12 enlaces unidireccionales, función de energía lineal, un conjunto de 4 niveles de energía y visibilidad proporcional. Cada enlace opera según un nivel de energía distinto dentro del rango $[0.05,1]$ donde con un valor de 0.05 el enlace está dormido y por tanto con un $5 \%$ de consumo de energía asociado, y con un valor de 1 el enlace estaría activo (operando a su capacidad nominal) con un $100 \%$ de consumo de energía.

\section{B. Regla de Transición}

Es el modelo estocástico que emplean las hormigas para tomar la decisión de elegir un nivel de energía u otro en el enlace que están explorando, permitiendo balancear entre la exploración de nuevos caminos y la explotación de los conocimientos acumulados hasta el momento. Para este proceso se usan dos tipos de información.

- Información memorística, que corresponde con el aporte o nivel de feromonas, y que mide la deseabilidad aprendida del movimiento. Dado un enlace $e \in E$, cada nivel de energía posible bajo el que pueda operar $n e_{e} \in N$ tendrá un aporte de feromonas $\tau_{\left(e, n e_{e}\right)} \in F$ donde $F$ es la matriz de feromonas. Este rastro de feromonas será modificado a lo largo de la ejecución del algoritmo aplicando la política de actualización y evaporación de las feromonas.

- Información heurística o alta visibilidad, que mide la preferencia heurística del movimiento, donde cada nivel de energía posible bajo el que pueda operar cada enlace tendrá una visibilidad $\eta_{\left(e, n e_{e}\right)} \in H$ donde $H$ es la matriz de visibilidad. $H$ tendrá unos valores iniciales y no modificables en la ejecución del algoritmo.

$$
P_{\left(e, n e_{e}\right)}^{k}= \begin{cases}\frac{\left[\tau_{\left(e, n e_{e}\right)}\right]^{\alpha} *\left[\eta_{\left(e, n e_{e}\right)}\right]^{\beta}}{\sum_{n e_{e} \in N_{e}}\left[\tau_{\left(e, n e_{e}\right)}\right]^{\alpha} *\left[\eta_{\left(e, n e_{e}\right)}\right]^{\beta}} & \text { si } n e_{e} \in N_{e} \\ 0 & \text { e.c.o. }\end{cases}
$$

La regla de transición se basa en la Eq. (4). Para una hormiga $k$ que esté ubicada en el enlace $e \in E$, el nivel de energía $n e_{e} \in N_{e}$ tendrá una probabilidad de ser seleccionado $P_{\left(e, n e_{e}\right)}^{k}$. Además se introduce el parámetro $\alpha$ que estima la importancia relativa de la información memorística o del camino recorrido en función del rastro de feromonas depositado y $\beta$ como la importancia de cercanía en términos de visibilidad o preferencia heurística.

\section{Actualización y Evaporación de las Feromonas}

La actualización de las feromonas se realiza mediante el Sistema de Hormigas-ciclo de ACS [15], [16], donde la deposición de las feromonas se lleva a cabo una vez que todas las hormigas de cada generación han obtenido una solución. Primero se produce un decaimiento o evaporación al valor de todos los rastros de feromonas, Eq. (5), con un factor constante $\gamma$ para evitar un incremento ilimitado de rastro de feromonas y para permitir olvidar las malas decisiones tomadas.

$$
\tau_{\left(e, n e_{e}\right)}=(1-\gamma) * \tau_{\left(e, n e_{e}\right)}
$$

Así, para el nivel de energía ne del enlace $e$, su valor asociado de rastro de feromonas será reducido una cantidad $\gamma \in(0,1]$. Posteriormente se realiza una retroalimentación positiva a los rastros de feromonas Eq. (6). Para ello se recorren todas las soluciones de las hormigas que han sido válidas $S V$ y se depositan feromonas a cada nivel de energía.

$$
\begin{aligned}
& \tau_{\left(e, n e_{e}\right)}=\tau_{\left(e, n e_{e}\right)}+\Delta \tau_{\left(e, n e_{e}\right)}^{k}, \forall n e_{e} \in s_{k}, \forall s_{k} \in S V \\
& \Delta \tau_{\left(e, n e_{e}\right)}^{k}=f\left(C\left(s_{k}\right)\right)=\frac{1}{C\left(s_{k}\right)}
\end{aligned}
$$

donde $\Delta \tau_{\left(e, n e_{e}\right)}^{k}$ es la cantidad de feromonas a retroalimentar, que depende de la calidad de la solución $C\left(s_{k}\right)$ de la hormiga $k$, al nivel de energía $n e_{e}$, que pertenece a la solución $s_{k}$.

$$
\tau_{\left(e, n e_{e}\right)}=\tau_{\left(e, n e_{e}\right)}+\Delta \tau_{\left(e, n e_{e}\right)}^{S}, \forall n e_{e} \in S
$$

Además, al aplicar EAS, se proporciona un peso adicional que refuercen los niveles de energía de aquellos enlaces que pertenecen a la mejor solución $S$ encontrada hasta el momento Eq. (7). 

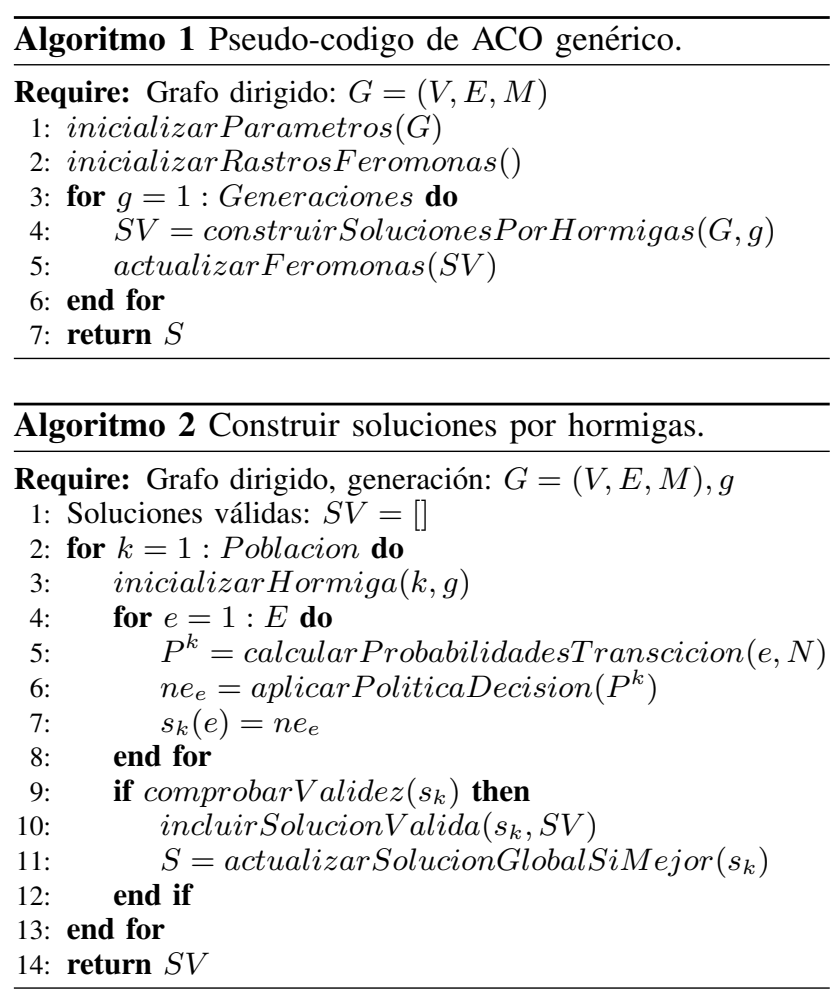

\section{Comprobar la Validez de una Solución}

Terminado el ciclo de construcción de la solución de una hormiga, es necesario comprobar su validez. Este proceso se realiza en dos pasos. Primero se comprueba que $\forall d_{(s, d)} \in M_{(i, j)}$, es posible encaminar todas las demandas de la matriz de tráfico dada aplicando Dijkstra [18] de modo que ninguno de los enlaces de la red se encuentra sobrecargado. Si la solución de la hormiga cumple dichos criterios de validación, se comparará con la mejor solución $S$ encontrada hasta el momento. En el caso de que la solución de la hormiga sea energéticamente más eficiente que $S$, la sustituirá como nueva mejor solución encontrada.

\section{E. Pseudocodigo ACO}

El pseudo-codigo del algoritmo ACO genérico puede observarse en el Algoritmo 1, mientras que en la Fig. 3 se presenta de forma gráfica como sería la ejecución de la primera generación del algoritmo ACO. Dada una topología de red y sus demandas de tráfico, se inicializan los parámetros del algoritmo ACO y las feromonas (Estado 1 de la Fig. 3). Además, se construyen tantas configuraciones de red (soluciones de hormigas) como tamaño tenga la población por cada una de las generaciones y se almacenan aquellas configuraciones válidas, $S V$. Posteriormente, se actualizan los niveles de feromonas mediante las soluciones válidas, $S V$, encontradas en la generación actual. Al final de la ejecución del algoritmo se obtiene la mejor configuración de red encontrada en términos de eficiencia energética.

El Algoritmo 2, representado por el Estado 2 de la Fig. 3, realiza el proceso de construcción de soluciones de las hormigas donde por cada enlace, aplica la regla de transición a los niveles de energía mediante los valores

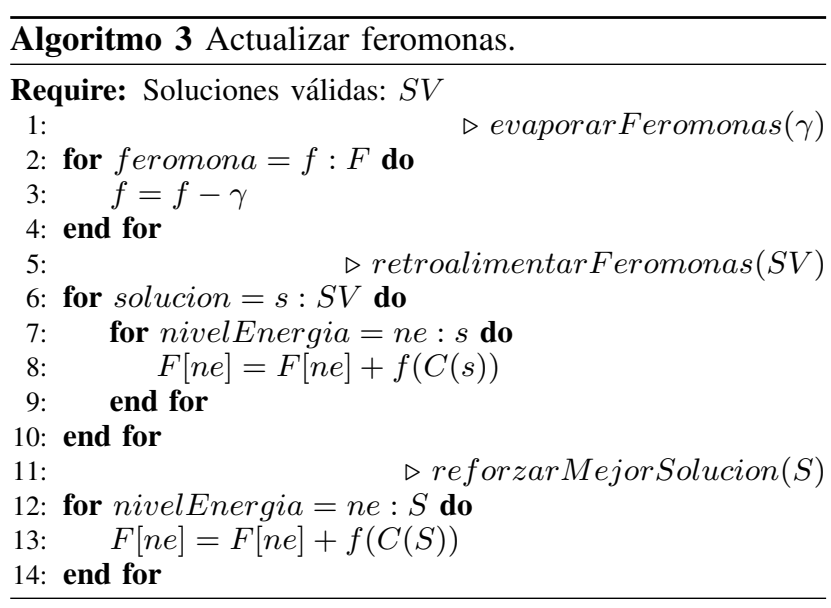

heurísticos y los rastros de feromonas, para obtener las probabilidades de elección de cada nivel de energía para el enlace. Posteriormente, con las probabilidades anteriores, aplica la política de decisión para seleccionar el definitivo nivel de energía para el enlace en cuestión (Estado 2.1 de la Fig. 3). Ponderados todos los enlaces con niveles de energía en la red, la hormiga devuelve $s$ como solución potencial. Por último, si esta configuración de red $s$ es válida, se compara con la mejor solución encontrada $S$ hasta el momento y, en el caso de que presente un menor consumo de energía, pasaría a convertirse en la mejor solución encontrada, $S$.

Por último, en el Algoritmo 3, Estado 3 de la Fig. 3, se desglosa el proceso de actualización de la matriz de feromonas. Primero se realiza el decaimiento de todos los rastros de feromonas mediante la constante $\gamma$. En el siguiente paso se realiza una retroalimentación positiva a aquellos rastros de feromonas que pertenezcan a las soluciones válidas para, finalmente, reforzar aquellos rastros de feromonas que pertenecen a la mejor solución encontrada.

\section{RESUltados EXPERIMENTALES}

En esta sección se analizan los resultados obtenidos tras realizar un conjunto de pruebas en las que se aplica el algoritmo ACO propuesto sobre topologías de red de distinto tamaño.

\section{A. Topologías y tráfico}

Con el fin de evaluar nuestra propuesta, se ha optado por tres escenarios de red reales: NSFNet (14 nodos y 42 elaces), Géant (23 nodos y 74 enlaces) y Germany50 (50 nodos y 176 enlaces), con una carga de tráfico entorno al 50\%. Tanto la información correspondiente a las topologías como las matrices de tráfico utilizadas se han extraído de [19].

\section{B. Parámetros y valores de ACO}

En la Tablas I se desglosan los parámetros utilizados por el algoritmo ACO para dar solución al problema de eficiencia energética en redes SDN. Se puede observar que se consideran dos tipos de matrices de visibilidad: Natural y Proporcional. A continuación se explican cada una de ellas. 
(1)

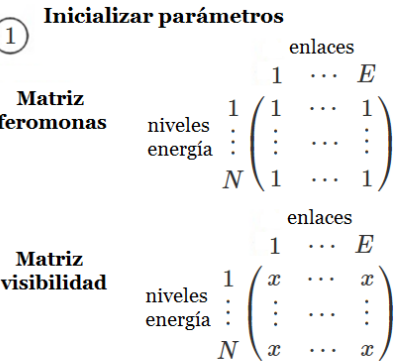

(3)

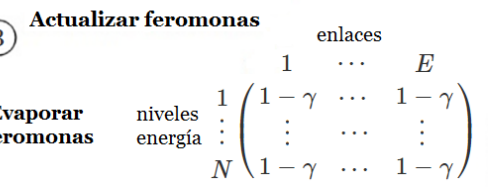

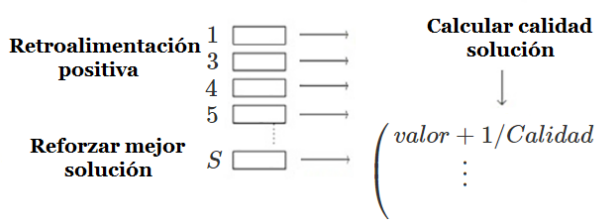

Fig. 3: Esquema del comportamiento del algoritmo ACO para el problema.
Tabla I: Parámetros de EEACO y valores considerados.

\begin{tabular}{|c|c|c|}
\hline Parámetro & Descripción & Valores \\
\hline$m$ & Tamaño de población & $20,50,80$ \\
\hline$g$ & Número de generaciones & $5,10,20$ \\
\hline$\alpha$ & $\begin{array}{l}\text { Importancia relativa de las } \\
\text { soluciones }\end{array}$ & 1 \\
\hline$\beta$ & $\begin{array}{l}\text { Importancia de cercanía en } \\
\text { términos de visibilidad }\end{array}$ & {$[2,5]$} \\
\hline$\gamma$ & Decaimiento de feromonas & $(0,1]$ \\
\hline$N$ & Niveles de energía & $2,3,4$ \\
\hline$H$ & Matriz de visibilidad & $\begin{array}{l}\text { Natural, Propor- } \\
\text { cional }\end{array}$ \\
\hline$F_{0}$ & Matriz de feromonas inicial & Todos a 1 \\
\hline$f$ & Función de energía & $\begin{array}{l}\text { Lineal, } \\
\text { Logarítmica }\end{array}$ \\
\hline$z$ & Repeticiones por prueba & 5 \\
\hline$T$ & Topologías consideradas & $\begin{array}{l}\text { NSFNet, Géant, } \\
\text { Germany50 }\end{array}$ \\
\hline
\end{tabular}

Para componer la matriz de visibilidad Natural se aplica Eq. (8), donde los valores heurísticos tendrán el mismo valor que el nivel de energía considerado.

$$
H_{\left(e, n e_{e}\right)}=n e_{e}
$$

En cambio, para componer la matriz de visibilidad Proporcional se aplica Eq. (9). En este caso, los valores heurísticos están comprendidos entre $[0.05,1]$. En la Fig. 4 se muestra un ejemplo de matrices de visibilidad obtenidas al aplicar las ecuaciones anteriores para una red compuesta por 5 enlaces que pueden operar según un conjunto de 4 niveles de energía.

$$
H_{\left(e, n e_{e}\right)}= \begin{cases}0.05 & \text { si } n e_{e}=1 \\ \left(\frac{1}{N-1}\right) *\left(n e_{e}-1\right) & \text { si } n e_{e}>1\end{cases}
$$

\section{Análisis de Resultados}

En esta sección se analizan los resultados obtenidos tras ejecutar el algoritmo ACO sobre los tres escenarios de red considerados. La metodología lleva a cabo para la obtención de resultados consiste en generar una muestra de 5 repeticiones de ejecución del algoritmo ACO por cada combinación de parámetros considerados en la Tabla I con un nivel de confianza del $95 \%$.

En las Figs. 5 y 6 se pueden observar los resultados obtenidos. En concreto, cada gráfica muestra los porcentajes de ahorro de energía obtenidos por el algoritmo ACO por cada función de energía y topología consideradas.

En la Fig. 5a se presentan los porcentajes de ahorro para las pruebas realizadas sobre la topología NSFNet con función de energía lineal. En ella, se puede observar que la visibilidad Natural se comporta mejor que la visibilidad Proporcional, llegando a unos valores máximos de ahorro energético cercanos al $25 \%$ para la visibilidad Natural, en comparación con el $20 \%$ de ahorro energético en el caso de la visibilidad Proporcional. Además, podemos observar que la mejor configuración en términos de eficiencia energética para la topología NSFNet con función de energía lineal, es considerar un conjunto de 4 niveles de energía y visibilidad Natural.

Para el caso de la función de energía logarítmica y la topología NSFNet, Fig. 6a, se pueden observar resultados similares a los obtenidos por las pruebas realizadas con función de energía lineal, es decir, la visibilidad Natural se comporta mejor que la visibilidad Proporcional. En cambio, si consideramos la función logarítmica, podemos observar que a mayor conjunto de niveles de energía, menor es el ahorro energético que se obtiene. Además, este comportamiento se repite para los dos tipos de visibilidad considerados. También cabe destacar que independientemente de la función de energía considerada, a 


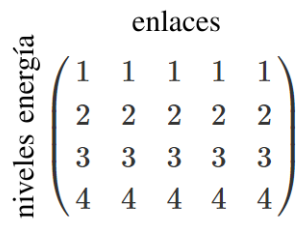

(a) Natural enlaces

\begin{tabular}{|c|c|c|c|c|c|}
\hline & \multicolumn{5}{|c|}{ enlaces } \\
\hline & 0.05 & 0.05 & 0.05 & 0.05 & 0.05 \\
\hline & 0.33 & 0.33 & 0.33 & 0.33 & 0.33 \\
\hline & 0.66 & 0.66 & 0.66 & 0.66 & 0.66 \\
\hline & 1 & 1 & 1 & 1 & 1 \\
\hline
\end{tabular}

(b) Proporcional

Fig. 4: Ejemplo matrices de visibilidad.

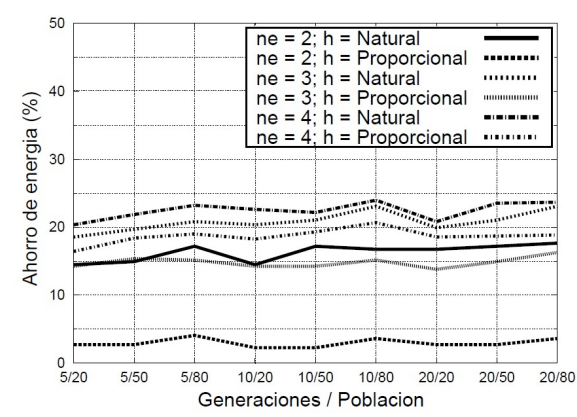

(a) NSFNet

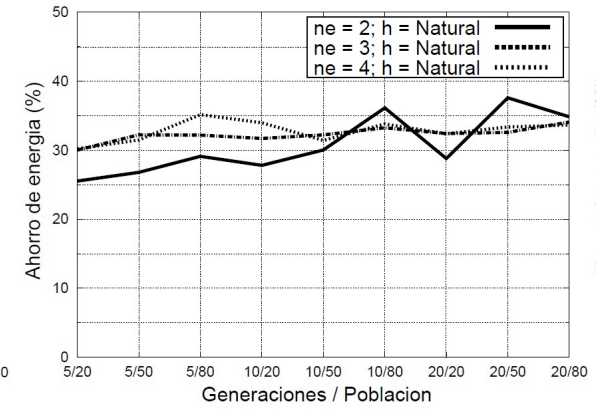

(b) Geant

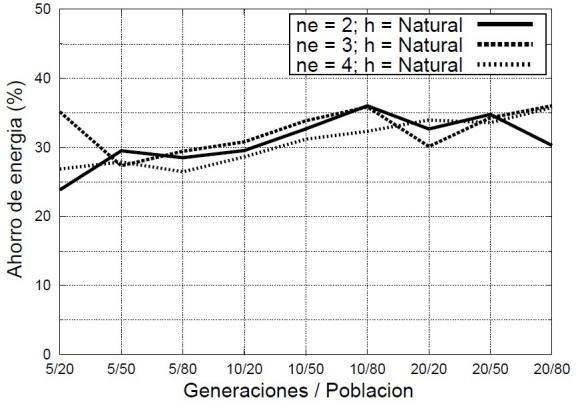

(c) Germany50

Fig. 5: Porcentaje de ahorro de energía en función del número de niveles de energía por enlace con función de energía lineal.

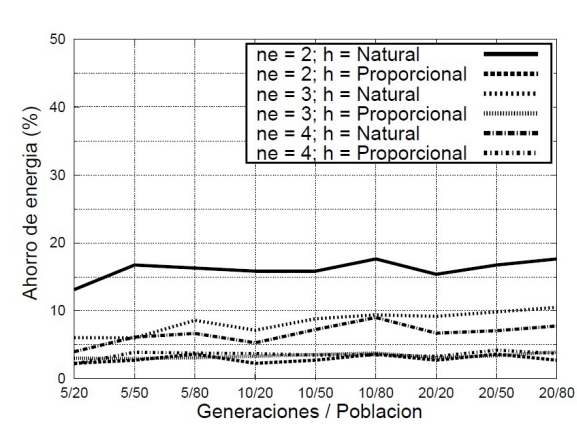

(a) NSFNet

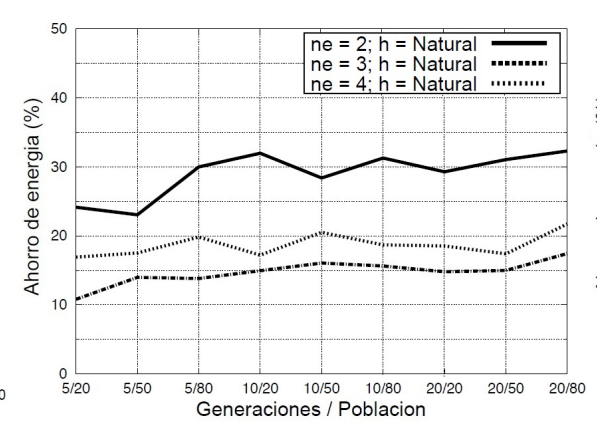

(b) Geant

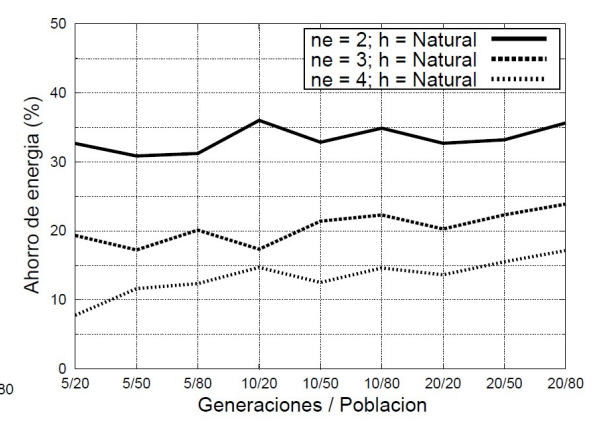

(c) Germany50

Fig. 6: Porcentaje de ahorro de energía en función del número de niveles de energía por enlace con función de energía logarítmica.

mayor número de generaciones y número de hormigas o población, mayor es el ahorro energético resultado del algoritmo ACO. Los resultados aplicando las topologías Géant y Germany50 tienen las mismas consideraciones que los anteriormente analizados para NSFNet, con la diferencia de que en estas dos topologías solamente se tiene en cuenta la visibilidad Natural, que es la visibilidad que mejor se ha comportado para la topología NSFNet. Por regla general, al considerar la función de energía lineal, a mayor conjunto de niveles de energía, mayor es el ahorro energético obtenido por el algoritmo ACO.

Por último, el tiempo de cómputo y procesamiento del algoritmo ACO es proporcional al número de generaciones y población tomadas como parámetros de entrada. Es decir, a mayor número de generaciones (iteraciones del algoritmo) y a mayor tamaño de la población, mayor será el tiempo de cómputo requerido. Otro factor que afecta directamente al tiempo de cómputo es el escenario de red considerado, ya que una topología de mayor tamaño tiene una repercusión operacional y temporal mayor. La relación de tiempo de cómputo de Germany50 es aproximadamente 12 veces superior al tiempo requerido para dar solución en la topología Géant y ésta, a su vez, es 7 veces superior a NSFNet. En la Tabla II se muestra un resumen de los resultados de las pruebas donde por cada topología se muestran los porcentajes de ahorro máximo y ahorro medio de consumo según la función de energía considerada para los enlaces.

Considerando los resultados obtenidos con la función de energía logarítmica podemos observar que a mayor 
Tabla II: Porcentajes de ahorro energético por topología.

\begin{tabular}{lcccc}
\hline \hline Topología & \% Max F.E.Lineal & \% Medio F.E.Lineal & \% Max F.E.Logarítmica & \% Medio F.E.Logarítmica \\
\hline NSFNet & 23,98 & 19,87 & 17,64 & 10,39 \\
Géant & 37,58 & 31,95 & 31,96 & 20,83 \\
Germany50 & 36,01 & 31,37 & 36,00 & 22,37 \\
\hline \hline
\end{tabular}

conjunto de niveles de energía menor es el ahorro energético obtenido. Este comportamiento radica en la propia definición de la función de energía logarítmica en comparación con la función de energía lineal, ya que habría que realizar una comparativa más justa de ambas funciones (Fig. 1) para que sus áreas delimitadoras con el eje de abscisas fueran iguales.

\section{Conclusiones y Trabajos Futuros}

En este trabajo se propone una solución al problema del consumo de energía en redes SDN. El algoritmo propuesto se basa en el comportamiento de las colonias de hormigas (ACO) para encontrar la configuración de red óptima en términos de eficiencia energética. Mediante la utilización del rastro de feromonas, las hormigas (o soluciones al problema) van creando soluciones potenciales en función del modo de operación activo de los enlaces (definidos en el artículo como niveles de energía). Además, se consideran dos funciones de energía diferentes: i) lineal o proporcional, tomada como base, donde a mayor velocidad de operación del enlace mayor consumo de energía; y ii) logarítmica, que simula el comportamiento del estándar EEE.

Tras realizar un conjunto de pruebas sobre topologías de red reales (NSFNet, Géant y Germany50), para las cuales varían los parámetros del algoritmo ACO, se obtienen un conjunto de resultados donde se puede observar que es posible conseguir un ahorro de energía significativo en torno al $30 \%$ dependiendo de la función de energía utilizada. La inclusión y aplicación del algoritmo propuesto no modificaría la arquitectura SDN tradicional, ya que únicamente bastaría con instalarlo en el controlador SDN de modo que se ejecute cada vez que se active un disparador (o trigger) en función de la superación de un umbral (como por ejemplo un incremento o decremento de tráfico significativo).

Como trabajo futuro, se plantea la posibilidad de evaluar el delay que puede producirse al cambiar de configuración de red en función del tráfico. Para ello, se podrían utilizar herramientas de emulación de redes SDN como Mininet y de generación de tráfico como iPerf.

\section{AgRADECIMIENTOS}

Este trabajo ha sido financiado, en parte, por el Ministerio de Economía, Industria y Competitividad (TIN201453986-REDT, TIN2015-67083-R y TIN2015-69957-R (MINECO/FEDER)), por el proyecto 4IE (0045-4IE-4-P) financiado por el Programa Interreg V-A España-Portugal (POCTEP) 2014-2020, por la Junta de Extremadura, Consejería de Economía e Infraestructuras (GR15098), y por el Fondo Europeo de Desarrollo Regional (FEDER).

\section{REFERENCIAS}

[1] E. Gelenbe and Y. Caseau, "The Impact of Information Technology on Energy Consumption and Carbon Emissions," Ubiquity, vol. 2015, no. June, pp. 1-15, 2015.

[2] L. Neves et al., "Smart 2020 report-Enabling the low carbon economy in the information age," The Climate Group, 2008.

[3] B. Aebischer and L. M. Hilty, "The Energy Demand of ICT: A Historical Perspective and Current Methodological Challenges. Springer" International Publishing, 2015.

[4] J. Chabarek, J. Sommers, P. Barford, C. Estan, D. Tsiang, and S. Wright, "Power awareness in network design and routing," in Proc. INFOCOM, Phoenix, AZ, USA, Apr. 2008, pp. 1130-1138.

[5] M. Gupta and S. Singh, "Greening of the Internet," in Proc. SIGCOMM, Karlsruhe, Germany, Aug. 2003, pp. 19-26.

[6] S. Nedevschi, L. Popa, G. Iannaccone, D. Wetherall, S. Ratnasamy, "Reducing network energy consumption via sleeping and rateadaptation,'Proc. 5th USENIX Symp. on Networked Systems Design and Implementation (NSDI '08), San Francisco, CA, 2008, pp. 323-336.

[7] F. Idzikowski, L. Chiaraviglio, A. Cianfrani, J. L. Vizcaíno, M Polverini, and Y. Ye, "A survey on energy-aware design and operation of core networks,'IEEE Commun. Surveys Tut., vol. 18, no. 2, pp. 1453-1499, 2nd Quart., 2015.

[8] L. Chiaraviglio, M. Mellia, F. Neri, "Reducing Power Consumption in Backbone Networks", Proc. 2009 IEEE Internat. Conf. on Communications (ICC 2009), Dresden, Germany, June 2009.

[9] S. M. Kerner, "Energy Efficient Ethernet hits standards milestone — InternetNews:The Blog — Sean Michael Kerner”. Internetnews blog. Último acceso 25/7/2017.

[10] J. Galán-Jiménez, A. Gazo-Cervero, ”Using bio-inspired algorithms for energy levels assessment in energy efficient wired communication networks, Journal of Network and Computer Applications, Vol 37, (2014) pp 171-185.

[11] D. Kreutz et al., "Software-defined networking: A comprehensive sur- vey," Proc. IEEE, vol. 103, no. 1, pp. 14-76, Jan. 2015.

[12] B. Heller, S. Seetharaman, P. Mahadevan, Y. Yiakoumis, P. Sharma, S. Banerjee, and N. McKeown, "Elastictree: Saving energy in data cen- ter networks." in NSDI, vol. 10, 2010, pp. 249-264.

[13] J. Wang, X. Chen, C. Phillips, and Y. Yan, "Energy efficiency with qos control in dynamic optical networks with sdn enabled integrated control plane," Computer Networks, vol. 78, pp. 57 - 67, 2015, special Issue: Green Communications.

[14] M. Dorigo, 1992. Optimization, Learning and Natural Algorithms, $\mathrm{PhD}$ thesis, Politecnico di Milano, Italy.

[15] M. Dorigo, V. Maniezzo \& A. Colorni, 1996. "Ant System: Optimization by a Colony of Cooperating Agents", IEEE Transactions on Systems, Man, and Cybernetics-Part B, 26 (1): 29-41.

[16] M. Dorigo, \& T. Stützle. (2003). The ant colony optimization metaheuristic: Algorithms, applications and advances. In Glover, F. \& Kochenberger, G. (Eds.), Handbook of Metaheuristics, 251285. Kluwer Academic Publishers.

[17] E. Bonabeau, M. Dorigo, G. Theraulaz (1999). Swarm Intelligence: From Natural to Artificial Systems. New York: Oxford University Press.

[18] E. W. Dijkstra (1959). A note on two problems in connexion with graphs. Numerische Mathematik 1: 269-271. doi:10.1007/BF01386390.

[19] SndLib. Data Source working Optimizaction of Telecommunications Networks. URL: http://sndlib.zib.de/home.action 